\title{
E-cadherin expression and cell proliferation in the primary tumor and metastatic lymph nodes of papillary thyroid microcarcinoma
}

\author{
MASANORI NAKAMURA, NAOYOSHI ONODA, SATORU NODA, SHINICHIRO KASHIWAGI, NAOKI AOMATSU, \\ KENTO KURATA, HIDEMI KAWAJIRI, TSUTOMU TAKASHIMA, TETSURO ISHIKAWA and KOSEI HIRAKAWA
}

Department of Surgical Oncology, Osaka City University Graduate School of Medicine, Osaka 545-8585, Japan

Received October 2, 2013; Accepted November 21, 2013

DOI: $10.3892 / \mathrm{mco} .2013 .220$

\begin{abstract}
Although papillary thyroid microcarcinoma (PTMC) has an excellent prognosis, certain cases exhibit aggressive clinical manifestations. In this study, we assessed the expression of E-cadherin and $\mathrm{Ki}-67$ in primary PTMC tumors and metastatic lymph nodes, in order to investigate the mechanism underlying the mainly indolent but potentially malignant nature of PTMC. A total of 93 PTMC patients treated in our institute were included in this study. All primary tumors and 57 metastatic lymph nodes were immunohistochemically stained and a total of 73 tumors (78.5\%) were positive for E-cadherin. E-cadherin expression was significantly less common at the invasive front $(58.1 \%, \mathrm{P}<0.01)$ compared to that at the center of the tumor. Tumors that had lost E-cadherin expression at the invasive front frequently presented with lymph node metastasis $(70.6 \%)$. Small tumors ( $\leq 5 \mathrm{~mm}$ diameter) expressed E-cadherin significantly more frequently compared with larger tumors $(\mathrm{P}=0.04)$; however, no other particular characteristic was found to correlate with the status of E-cadherin expression in the primary tumors. E-cadherin expression was detected in $49(86.0 \%)$ of the 57 metastatic foci and correlated significantly with the expression status at the invasive front of the tumor $(\mathrm{P}=0.02)$. The Ki-67 index was universally low and was not correlated with the clinicopathological characteristics or the E-cadherin expression of the tumors. These results suggested that cancer cells in the metastatic lymph nodes exhibit indolent characteristics, similar to those of the primary PTMC. However, the metastatic cancer cells may have already completed the process of epithelial-to-mesenchymal transition (EMT) and
\end{abstract}

Correspondence to: Associate Professor Naoyoshi Onoda, Osaka City University Graduate School of Medicine, 1-4-3 Asahi-machi, Abeno-ku, Osaka 545-8585, Japan

E-mail: nonoda@med.osaka-cu.ac.jp

Key words: papillary thyroid microcarcinoma, E-cadherin, lymph node metastasis, epithelial-to-mesenchymal transition, mesenchymal-to-epithelial transition mesenchymal-to-epithelial transition (MET), suggesting an innate malignant potential.

\section{Introduction}

According to the World Health Organization classification system for thyroid tumors, papillary thyroid microcarcinoma (PTMC) is defined as a papillary thyroid carcinoma (PTC) measuring $\leq 10 \mathrm{~mm}$ in its greatest dimension (1). The occurrence of PTMC is on the increase worldwide (2-4), along with the frequent application of neck ultrasonography (US) $(5,6)$. PTMC is generally characterized by an indolent clinical course and has an excellent prognosis, with a disease-specific mortality of $<0.5 \%$ (6). However, certain PTMC cases were found to be clinically aggressive $(6,7)$. According to the observations of Sugitani et al (7), PTMC patients with bulky lymph node metastases or extrathyroidal invasion were at the highest risk for a cancer-specific fatal outcome. Those observations suggested that there is a group of PTMCs that have already acquired highly malignant potential. However, the basic mechanism underlying the development of aggressive characteristics in these tumors has not yet been identified.

The reported risk factors for PTMC recurrence were shown to be male gender $(8,9)$, extent of primary surgery $(10)$, presence of lymph node metastases at initial diagnosis $(3,8-12)$, tumor multifocality $(3,8,12)$ and capsular invasion $(9,11-14)$. Furthermore, male gender, tumor multifocality and capsular invasion are considered to be risk factors characteristic of lymph node metastasis $(13,15)$. Several studies reported that the presence of clinical lymph node metastasis in PTMC was one of the most important prognostic indicators, whereas others demonstrated that pathological lymph node metastasis identified following prophylactic dissection did not significantly affect patient prognosis. Pathological lymph node metastasis from PTMC was found in a range of 26-56\% (15-20). The reason(s) for the difference between clinical and pathological lymph node metastasis have not been fully elucidated.

In Japan, thyroid function-preserving surgery with prophylactic lymph node dissection is considered to be the standard procedure for PTMC. Postoperative follow-up with surgeon-performed US, without radioactive iodine ablation (RIA) or thyroid-stimulating hormone (TSH) suppression, is widely 
applied as the standard management, due to the strict regulations on radioisotope use, the shortage of institutes that perform radioiodine therapy and the difficulties in restricting iodine intake in the daily diet (21). Therefore, we were able to enroll a number of PTMC patients who were diagnosed with pathological lymph node metastasis, with a long follow-up period.

The required steps for cancer cells to form metastases are escape from the primary tumor, active migration toward the vasculature and survival within the systemic circulation. To successfully undertake these steps, cancer cells may alter their characteristics from an epithelial- to a mesenchymal-like form (epithelial-to-mesenchymal transition; EMT) (22). E-cadherin is a well-known cellular adhesion molecule in epithelial cells and is known to be lost during the process of EMT. E-cadherin expression is commonly observed in differentiated thyroid cancer (23-27) and loss of its expression was reported to be an independent prognostic factor for these tumors (27).

In the present study, we investigated the expression of E-cadherin and Ki-67-index (markers for EMT and cell proliferation, respectively) in PTMC cases. The results demonstrated that loss of E-cadherin expression is correlated with lymph node metastasis, although re-expression of E-cadherin was commonly detected in the metastatic foci, without observed acceleration of proliferation, suggesting that the metastatic cancer cells in the lymph nodes exhibit basically indolent characteristics, with an innate malignant potential.

\section{Patients and methods}

Patients. A consecutive series of 93 patients with PTMC who were surgically treated in our institute between 2000 and 2010 was investigated (Table I). The patients with incidental cancer found following surgery or concomitant multiple lesions $>1 \mathrm{~cm}$ in diameter were excluded from this study. All patients were diagnosed with PTC prior to surgery by fine-needle aspiration cytology. The postoperative pathological examination confirmed the absence of a poorly differentiated carcinoma component. The cases included 10 male and 83 female patients, with a median age of 55 years (range, 24-80 years). A total of 66, 5 and 22 patients underwent lobectomy, subtotal, or total thyroidectomy, respectively. All patients underwent lymph node dissection, including at least the central compartment. A total of 17 patients underwent therapeutic lateral lymph node dissection. Ipsilateral and bilateral compartment dissection was performed in 72 and 9 patients, respectively. Pathological nodal involvement was detected in $57(61.3 \%)$ of the patients, 26 patients $(28.0 \%)$ had nodal metastasis in the central compartment only and 31 (33.3\%) had metastatic lymph nodes in the lateral compartment.

RIA and TSH suppression were performed in 11 and 10 patients, respectively, including 1 patient with lung metastasis, whereas 6 patients received both treatments. All patients were followed-up at our institute for 30-136 months (median, 78 months) by annual US and thyroid function tests. Iodine scintigraphy or computed tomography were performed when considered necessary. Two patients had a recurrence in the lymph nodes at the ipsilateral submandibular compartment and required additional dissection. No other patients developed lymph node or distant metastases.
Table I. Characteristics of the study population: patients with papillary microcarcinoma investigated.

\begin{tabular}{lcr}
\hline Category & No. of patients & $\%$ \\
\hline Gender (male:female) & $10: 83$ & - \\
Age at operation, years & 24-80; median, 55 & - \\
Extent of surgery & & \\
Lobectomy & 66 & 71.0 \\
Subtotal Tx & 5 & 5.4 \\
Total Tx & 22 & 23.7 \\
Extent of dissection & & \\
Central & 12 & 12.9 \\
Lateral & & \\
$\quad$ Ipsilateral & 72 & 9.7 \\
$\quad$ Bilateral & 9 & \\
\hline
\end{tabular}

Tx, thyroidectomy.

Immunohistochemistry. The 93 primary tumors and the metastatic lesions in the lymph nodes from 57 patients were immunohistochemically stained as described previously (28). Briefly, the specimens were fixed in $10 \%$ formaldehyde solution and embedded in paraffin. The specimens were then cut in 4- $\mu \mathrm{m}$ sections and deparaffinized in xylene. The tissues were heated for $20 \mathrm{~min}$ at $105^{\circ} \mathrm{C}$ by autoclave in Target Retrieval solution (Dako, Carpinteria, CA, USA). Following blocking of the endogenous peroxidase activity, the sections were incubated in normal goat serum. The M3612 and M7240 primary antibodies (DakoCytomation, Glostrup, Denmark) were used to detect E-cadherin and $\mathrm{Ki}-67$, respectively. The tissue sections were incubated with each antibody for $60 \mathrm{~min}$ at room temperature or overnight at $4^{\circ} \mathrm{C}$, followed by incubation with a secondary antibody and treatment with streptavidin-peroxidase reagent [Histofine SAB-PO (M) kit; Nichirei, Tokyo, Japan]. Diaminobenzidine was added as a chromogen, followed by counterstaining with Mayer's hematoxylin.

The specific immune reactivity of E-cadherin was detected on the membranous surface of the cancer cells. The membranous staining of the follicular cells in adjacent normal thyroid tissue was used as positive control. Positive staining was defined as $>10 \%$ of the cancer cells showing specific immune reactivity at the center of the tumor. Seven tumors were surrounded by a thick fibrous capsule and the immune reactivity of E-cadherin at the invasive front was investigated in the remaining 86 tumors. The invasive front was defined as the interface $(<0.1 \mathrm{~cm})$ between the tumor and the adjacent non-neoplastic tissue (29).

The Ki-67 index was defined as the percentage of cancer cells with positive nuclear staining for Ki-67.

Statistical analysis. Statistical analysis was performed using SPSS 13.0 statistical software (SPSS, Inc., Chicago, IL, USA). The Chi-squared test was used to compare the differences in the positivity rate for immunostaining and the clinicopathological factors and $\mathrm{P}<0.05$ was considered to indicate a statistically significant difference. 
Table II. Association of clinicopathological characteristics of the patients with E-cadherin expression and Ki-67 index of the tumor.

E-cadherin expression

Characteristics

$(-) \quad(+)$

$\%$

P-value

Ki-67 index (\%)

Gender

Male

n

$(+)$

(0.0

Female

10

1

9

90.0

0.317

1.19

Age at operation, years

83

19

64

77.1

1.28

$<45$

\begin{tabular}{l}
$<45$ \\
\hline
\end{tabular}

23

7

16

57

69.6

0.230

1.44

13

81.4

1.21

Tumor diameter, $\mathrm{mm}$

6-10

12

81

0

12

61

100.0

0.044

1.67

75.3

1.29

Capsular invasion

$\begin{array}{ll}\text { Negative } & 5 \\ \text { Positive } & 37 \\ \mathrm{pT} & \\ 1 \mathrm{a} & 5 \\ 3 & 35 \\ 4 & \\ \mathrm{pN} & \\ 0 & 3 \\ 1 \mathrm{a} & \\ 1 \mathrm{~b} & \\ \mathrm{M} & 3 \\ 0 & \\ 1 & \end{array}$

$56 \quad 13$

43

76.8

0.622

1.39

37

7

30

81.1

1.05

$\begin{array}{lll}56 & 13 & 43\end{array}$

43
28

76.8

0.622

1.39

80.0

1.07

7

100.0

0.50

$\begin{array}{lll}36 & 9 & 27\end{array}$

$\begin{array}{lll}36 & 6 & 20\end{array}$

75.0

0.514

1.31

76.9

1.74

83.9

0.88

1

26

92

20

72

78.3

100.0

pStage

I

II

III

$\begin{array}{rr}39 & 11 \\ 1 & \\ 29 & 7 \\ 24 & 2\end{array}$

11
0
7
2

28

71.8

$\begin{array}{rr}1 & 100.0 \\ 22 & 75.9\end{array}$

0.785

1.27

91.7

1.24

1.63

0.89

E-cadherin

Negative

Positive

Total

93

20

73 $2.27\left(1.00^{\mathrm{a}}\right)$

1.09

1.27

${ }^{a}$ When a case scored $15 \%$ it was excluded.

\section{Results}

A total of 73 of the 93 tumors $(78.5 \%)$ exhibited specific membranous staining for E-cadherin (Fig. 1). E-cadherin expression was detected in the 12 smaller tumors $(\leq 5 \mathrm{~mm})$ and was significantly more commonly expressed in the smaller compared with the 81 larger tumors. There was no observed correlation between E-cadherin expression and gender, age, or other pathological factors, including nodal involvement (Table II).

A total of 17 (25.4\%) of the 67 evaluable E-cadherin-positive tumors had lost E-cadherin expression at the invasive front. By contrast, no E-cadherin-negative tumor gained its expression at the invasive front. Therefore, E-cadherin expression was significantly less commonly found at the invasive front compared to the center of the tumor $(58.1 \%, \mathrm{P}<0.01$, Table III). Of the 17 patients with tumors that had lost E-cadherin expression at the invasive front, $12(70.6 \%)$ presented with nodal involvement. This frequency of nodal involvement was higher compared to that in cases that retained E-cadherin expression at the invasive front $(28 / 50 ; 56.0 \%)$ or had not expressed E-cadherin in the primary tumor $(11 / 19 ; 57.9 \%)$.

E-cadherin expression was frequently found in the metastatic foci in the involved lymph nodes (49 of 57 nodes, $86.0 \%)$. Ten of the 11 cases with E-cadherin-negative tumors exhibited E-cadherin expression in the metastatic foci in the 
Table III. E-cadherin expression in the tumor and comparison of expression at the center with that at the invasive front of the tumor.

\begin{tabular}{lccccc}
\hline & \multicolumn{2}{c}{ At the invasive front } & & & \\
At the center & Negative (\%) & Positive (\%) & Subtotal (\%) & Encapsulated tumors & Total (\%) \\
\hline Negative & 19 & 0 & 19 & 1 & $20(21.5)$ \\
Positive & 17 & 50 & 67 & 6 & $73(78.5)$ \\
Total & $36(41.9)$ & $50(58.1)$ & $86(100)$ & 7 & $93(100)$ \\
\hline
\end{tabular}

Table IV. Correlation between E-cadherin expression in the tumor and that in the metastatic foci in the involved lymph nodes.

\begin{tabular}{lcccr}
\hline & \multicolumn{2}{c}{ In the metastatic lymph nodes } & & \\
\cline { 2 - 3 } In the primary tumor & Negative $(\%)$ & Positive $(\%)$ & Total $(\%)$ & P-value \\
\hline At the center & $8(14.0)$ & $49(86.0)$ & $57(100)$ & 0.538 \\
Negative & 1 & 10 & $11(19.3)$ & $46(80.7)$ \\
Positive & 7 & 39 & $57(100)$ & 0.024 \\
At the invasive front & $8(14.0)$ & $49(86.0)$ & $23(40.3)$ & \\
Negative & 6 & 17 & $29(50.9)$ & $5(8.8)$ \\
Positive & 1 & 28 & 4 & \\
Encapsulated & 1 & 4 & \\
\hline
\end{tabular}

Table V. Correlation between loss of E-cadherin expression at the invasive front of the tumor and at the metastatic foci in the involved lymph nodes.

\begin{tabular}{|c|c|c|c|c|}
\hline \multicolumn{2}{|c|}{ In the primary tumor } & \multicolumn{2}{|c|}{ In the metastatic lymph nodes } & \multirow[b]{2}{*}{ Total $(\%)$} \\
\hline At the center & At the invasive front & Negative (\%) & Positive (\%) & \\
\hline \multirow[t]{2}{*}{ Negative } & Negative & 1 & 10 & $11(19.3)$ \\
\hline & Positive & 0 & 0 & 0 \\
\hline \multirow[t]{3}{*}{ Positive } & Negative & $5^{\mathrm{a}}$ & 7 & $12(21.1)$ \\
\hline & Positive & 1 & 28 & $29(50.9)$ \\
\hline & Encapsulated & 1 & 4 & $5(8.8)$ \\
\hline Total & & $8(41.9)$ & $49(58.1)$ & $57(100)$ \\
\hline
\end{tabular}

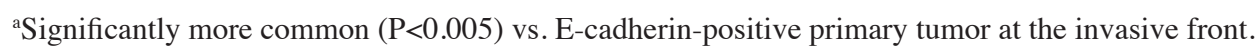

involved lymph nodes. There was no correlation between the E-cadherin expression status at the center of the primary tumor and that at the metastatic foci. However, there was a positive correlation between the E-cadherin expression status at the invasive front and that at the metastatic foci $(\mathrm{P}=0.024$, Table IV). A total of 8 lymph node metastatic foci did not express E-cadherin. Although one of these lesions recurred in the neck, no remarkable clinical characteristics were observed in the other cases. Five of these 8 cases (62.5\%) exhibited loss of E-cadherin expression at the invasive front of the primary tumor. The rate of E-cadherin-negative metastatic foci was significantly higher among cases in which the tumor had lost E-cadherin expression at the invasive front compared to those in which its expression at the invasive front of the primary tumor was retained (Table $\mathrm{V}, \mathrm{P}<0.005$ ).
The Ki-67 index ranged between 0 and $15 \%$ in the tumors, with only one primary tumor scoring $>5 \%$. Thus, the Ki-67 index was almost universally $\leq 5 \%$ in the tumors and did not demonstrate any significant differences according to the clinicopathological characteristics of the patients or the E-cadherin expression of the tumors (Table II). There was no difference in the Ki-67 index at the invasive front (mean \pm standard deviation, $1.03 \pm 0.86 \%$ ) compared to that at the center of the tumor $(2.27 \pm 4.29 \%)$. The Ki-67 index in the metastatic foci was universally $\leq 3 \%(0.83 \pm 0.83 \%)$ (Fig. 2). Two cases that developed recurrence during the follow-up period did not exhibit high Ki-67 indices $(<1 \%)$. The only patient with a Ki-67 index of $15 \%$ had a single $6 \mathrm{~mm}$-diameter tumor, without capsular invasion (T1a, N1a, M0, stage III) and remains alive and disease-free for $>5$ years. 


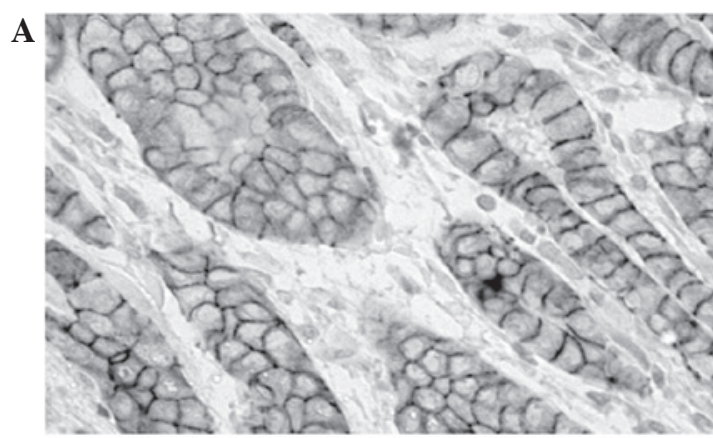

B
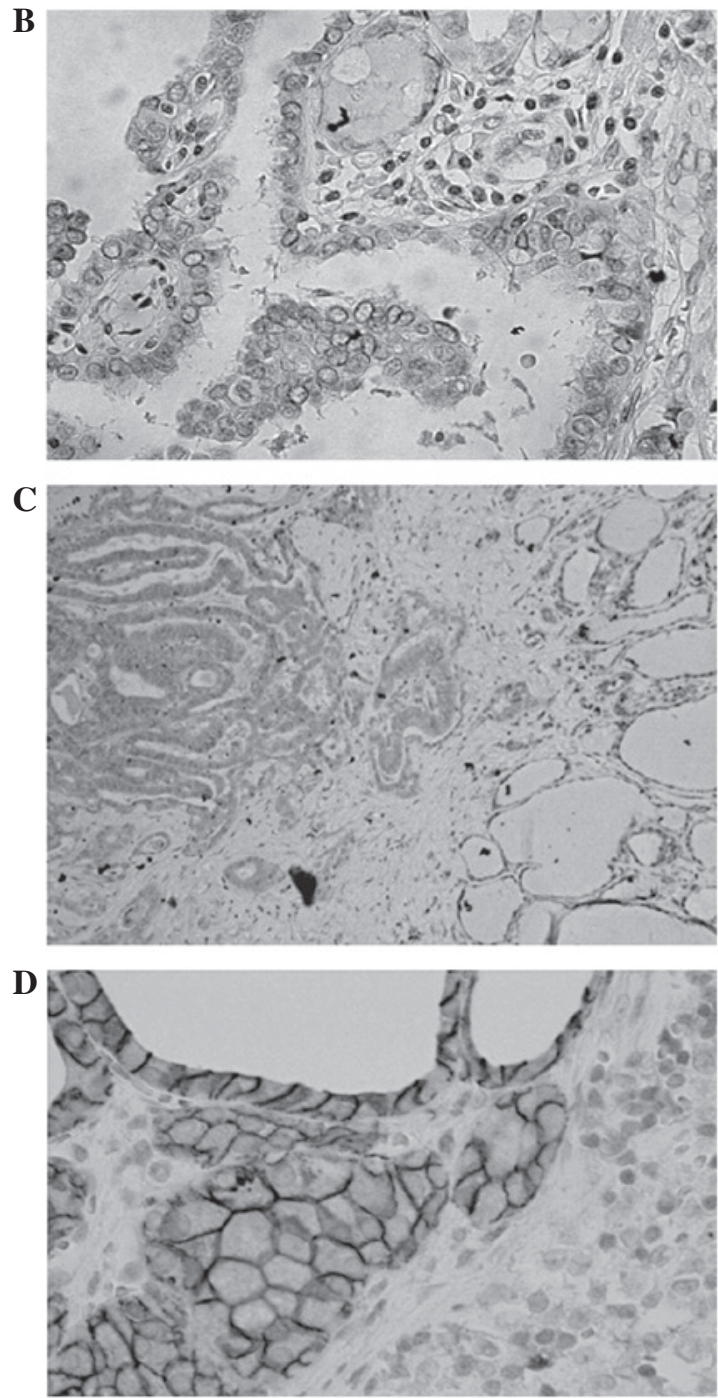

Figure 1. Representative results of E-cadherin immunostaining (A) E-cadherin was commonly found to be expressed on the membranous surface of the cancer cells of papillary thyroid microcarcinoma. (B) Approximately $20 \%$ of the cases were negative for E-cadherin expression. (C) E-cadherin expression was diminished at the invasive front in one-fourth of the E-cadherin-positive tumors. (D) E-cadherin was frequently expressed in the metastatic lesions in the lymph nodes.

\section{Discussion}

Total thyroidectomy followed by RIA is often performed as initial treatment for patients with PTMC $(8,30)$. However, there has been accumulating information justifying a less invasive treatment for PTMC, due to its excellent prognosis following less extensive surgery $(4,16,17,31-33)$, or even observation without

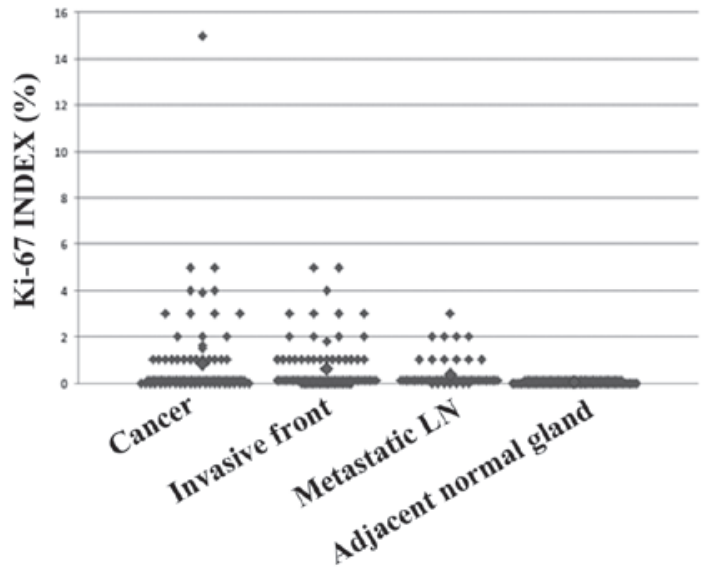

Figure 2. Ki-67 index according to the site of investigation. The Ki-67 index was universally low. LN, LN, lymph node.

surgery $(34,35)$. Indeed, only a few fatal PTMC cases have been recorded among a small proportion of high-risk patients. The optimal management strategy for patients with PTMC should be further investigated $(32,36)$, as there is lack of sufficient information regarding the basic characteristics of PTMC.

In the present study, we detected frequent and stable expression of E-cadherin in PTMCs. These results are in accordance with those of previous studies investigating E-cadherin expression in PTCs of all stages (23-27). Certain studies demonstrated a correlation between the loss of E-cadherin expression and disease progression or poorer prognosis in PTC $(27,29)$; however, the results of the present study did not verify the clinical significance of E-cadherin expression in PTMC. The majority of PTMCs exhibited low $\mathrm{Ki}-67$ indices, suggesting a low level of proliferation. There were no cases with fatal outcome or highest-risk factors for cancer-specific mortality. However, it may be necessary to include aggressive PTMC cases in future studies, in order to determine the clinical significance of the loss of E-cadherin expression, although such cases are rare.

In a quarter of the cases with E-cadherin-positive tumors included in the present study, we observed a decrease in E-cadherin expression at the invasive front. This phenomenon was also reported by Liu et al (29) in larger PTCs. The authors of that study also demonstrated that E-cadherin loss was associated with a loss of cohesiveness and polarity, which was similar to our findings. These phenotypical and morphological changes are characteristic of EMT (29). Furthermore, we observed that the tumors that had lost E-cadherin expression at the invasive front, commonly presented with lymph node involvement, suggesting clinical characteristics of EMT. It is therefore indicated that, even in small PTMCs, the process of cancer cell dispersion has already been initiated and parts of the tumor may have acquired aggressive characteristics.

It was also observed that E-cadherin was frequently re-expressed in the cancer cells that formed metastatic foci in the involved lymph nodes. A similar observation was described as the 're-expression of E-cadherin' in a previous study investigating PTC and follicular carcinoma of all stages (27). According to recent findings, this re-expression step of E-cadherin is considered to represent a 
mesenchymal-to-epithelial transformation (MET), the reversal of EMT. This phenotypical reversal is considered to be a step toward creating suitable conditions for cancer cells under which they may survive and form metastatic foci within an environment other than that of the primary site (37). Re-expression of E-cadherin in metastatic lymph nodes was commonly observed in patients with E-cadherin-negative primary PTMC in the present study, suggesting that MET is crucial for the formation of metastatic lesions. There was no upregulation of proliferative activity at the metastatic site, as demonstrated by the low Ki-67 indices in these cancer cells, indicating that the majority of the PTMC metastatic foci in the involved lymph nodes exhibited an indolent phenotype, similar to that of the primary site.

The loss of E-cadherin expression was one of several profiles that indicated phenotypical characteristics suggesting EMT in PTC (38). Several other factors are also known to be involved in this process (39). The expression of transforming growth factor- $\beta$ (TGF- $\beta$ ) in PTMC was shown to be associated with high proliferative potential and clinically aggressive characteristics (14). A previous study by Knauf et al (40) suggested that PTC with B-raf mutation has the potential to acquire aggressive characteristics through EMT by the stimulation of TGF- $\beta$. B-raf mutations are frequently found in PTMCs and are associated with distinctive morphology and aggressive behaviour $(12,41)$.

It is hypothesized that a certain type of stimulation may irreversibly alter the phenotype of cancer cells when they have already acquired the ability to transform. RIA may be effective in eradicating cancer cells in metastatic lymph nodes, due to their characteristics regarding iodine uptake (42). However, the indication and efficacy of RIA in low-risk PTMC cases remains questionable $(4,43)$. We still need to determine how indolent PTMC cells respond to stimulation inducing cell proliferation, by TSH or other factors that induce EMT, prior to the application of RIA in low-risk patients.

In conclusion, this study demonstrated that cancer cells in the metastatic lymph nodes exhibited indolent characteristics, similar to those of the primary PTMC. However, the metastatic cancer cells may have already completed the processes of EMT and MET, suggesting an innate malignant potential. Further studies are required to identify the trigger that initiates the change of indolent PTMC cancer cells to the aggressive phenotype.

\section{Acknowledgements}

This study was supported in part by a Grant-in-Aid for Scientific Research (Kakenhi no. 25461992).

\section{References}

1. LiVolsi VA, Albores-Saavedra J, Asa SL, et al: Papillary carcinoma. In: World Health Organization Classification of Tumours: Pathology and Genetics. Tumours of the Endocrine Organs. De Lellis RA, Lloyd RV, Heitz PU and Eng C (eds) Vol 8. IARC Press International Agency for Research on Cancer, Lyon, pp57-66, 2004

2. Leenhardt L, Grosclaude P and Chérié-Challine L; Thyroid Cancer Committee: Increased incidence of thyroid carcinoma in France: a true epidemic or thyroid nodule management effects? Report from the French Thyroid Cancer Committee. Thyroid 14 1056-1060, 2004.
3. Chow SM, Law SC, Chan JK, et al: Papillary microcarcinoma of the thyroid - Prognostic significance of lymph node metastasis and multifocality. Cancer 98: 31-40, 2003.

4. Hay ID, Hutchinson ME, Gonzalez-Losada T, et al: Papillary thyroid microcarcinoma: a study of 900 cases observed in a 60-year period. Surgery 144: 980-988, 2008.

5. Karatzas T, Vasileiadis I, Kapetanakis S, et al: Risk factors contributing to the difference in prognosis for papillary versus micropapillary thyroid carcinoma. Am J Surg 206: 586-593, 2013.

6. RotiE, degli UbertiEC, Bondanelli Mand Braverman LE: Thyroid papillary microcarcinoma: a descriptive and meta-analysis study. Eur J Endocrinol 159: 659-673, 2008.

7. Sugitani I, Toda K, Yamada K, et al: Three distinctly different kinds of papillary thyroid microcarcinoma should be recognized: our treatment strategies and outcomes. World J Surg 34: 1222-1231, 2010.

8. Buffet C, Golmard JL, Hoang C, et al: Scoring system for predicting recurrences in patients with papillary thyroid microcarcinoma. Eur J Endocrinol 167: 267-275, 2012.

9. Riss JC, Peyrottes I, Chamorey E, et al: Prognostic impact of tumour multifocality in thyroid papillary microcarcinoma based on a series of 160 cases. Eur Ann Otorhinolaryngol Head Neck Dis 129: 175-178, 2012.

10. Hay ID, Grant CS, van Heerden JA, et al: Papillary thyroid microcarcinoma: a study of 535 cases observed in a 50 -year period. Surgery 112: 1139-1147, 1992.

11. Pisanu A, Reccia I, Nardello O and Uccheddu A: Risk factors for nodal metastasis and recurrence among patients with papillary thyroid microcarcinoma: differences in clinical relevance between nonincidental and incidental tumors. World J Surg 33: 460-468, 2009

12. Zheng X, Wei S, Han Y, et al: Papillary microcarcinoma of the thyroid: clinical characteristics and BRAF(V600E) mutational status of 977 cases. Ann Surg Oncol 20: 2266-2273, 2013.

13. Gulben K, Berberoglu U, Celen O and Mersin HH: Incidental papillary microcarcinoma of the thyroid - factors affecting lymph node metastasis. Langenbecks Arch Surg 393: 25-29, 2008.

14. Sugitani I, Yanagisawa A, Shimizu A, Kato M and Fujimoto Y: Clinicopathologic and immunohistochemical studies of papillary thyroid microcarcinoma presenting with cervical lymphadenopathy. World J Surg 22: 731-737, 1998.

15. Zhang L, Wei WJ, Ji QH, et al: Risk factors for neck nodal metastasis in papillary thyroid microcarcinoma: a study of 1066 patients. J Clin Endocrinol Metab 97: 1250-1257, 2012.

16. Hyun SM, Song HY, Kim SY, et al: Impact of combined prophylactic unilateral central neck dissection and hemithyroidectomy in patients with papillary thyroid microcarcinoma. Ann Surg Oncol 19: 591-596, 2012.

17. Caliskan M, Park JH, Jeong JS, et al: Role of prophylactic ipsilateral central compartment lymph node dissection in papillary thyroid microcarcinoma. Endocr J 59: 305-311, 2012.

18. Zeng RC, Li Q, Lin KL, et al: Predicting the factors of lateral lymph node metastasis in papillary microcarcinoma of the thyroid in eastern China. Clin Transl Oncol 14: 842-847, 2012.

19. Zhou YL, Gao EL, Zhang W, et al: Factors predictive of papillary thyroid micro-carcinoma with bilateral involvement and central lymph node metastasis: a retrospective study. World J Surg Oncol 10: 67, 2012.

20. Kim BY, Jung CH, Kim JW, et al: Impact of clinicopathologic factors on subclinical central lymph node metastasis in papillary thyroid microcarcinoma. Yonsei Med J 53: 924-930, 2012.

21. Sugitani I and Fujimoto Y: Management of low-risk papillary thyroid carcinoma: unique conventional policy in Japan and our efforts to improve the level of evidence. Surg Today 40: 199-215, 2010.

22. Kalluri R and Weinberg RA: The basics of epithelial-mesenchymal transition. J Clin Invest 119: 1420-1428, 2009.

23. Batistatou A, Charalabopoulos K, Nakanishi Y, et al: Differential expression of dysadherin in papillary thyroid carcinoma and microcarcinoma: correlation with E-cadherin. Endocr Pathol 19: 197-202, 2008.

24. Choi YL, Kim MK, Suh JW, et al: Immunoexpression of HBME-1, high molecular weight cytokeratin, cytokeratin 19 , thyroid transcription factor-1, and E-cadherin in thyroid carcinomas. J Korean Med Sci 20: 853-859, 2005.

25. Naito A, Iwase H, Kuzushima T, Nakamura T and Kobayashi S: Clinical significance of E-cadherin expression in thyroid neoplasms. J Surg Oncol 76: 176-180, 2001.

26. Kapran Y, Ozbey N, Molvalilar S, et al: Immunohistochemical detection of E-cadherin, alpha- and beta-catenins in papillary thyroid carcinoma. J Endocrinol Invest 25: 578-585, 2002. 
27. von WasielewskiR,Rhein A,WernerM,etal:Immunohistochemical detection of E-cadherin in differentiated thyroid carcinomas correlates with clinical outcome. Cancer Res 57: 2501-2507, 1997.

28. Kashiwagi S, Yashiro M, Takashima T, et al: c-Kit expression as a prognostic molecular marker in patients with basal-like breast cancer. Br J Surg 100: 490-496, 2013.

29. Liu Z, Kakudo K, Bai Y, et al: Loss of cellular polarity/cohesiveness in the invasive front of papillary thyroid carcinoma, a novel predictor for lymph node metastasis; possible morphological indicator of epithelial mesenchymal transition. J Clin Pathol 64: 325-329, 2011

30. Pelizzo MR, Boschin IM, Toniato A, et al: Natural history, diagnosis, treatment and outcome of papillary thyroid microcarcinoma (PTMC): a mono-institutional 12-year experience. Nucl Med Commun 25: 547-552, 2004.

31. Lee J, Park JH, Lee CR, Chung WY and Park CS: Long-term outcomes of total thyroidectomy versus thyroid lobectomy for papillary thyroid microcarcinoma: comparative analysis after propensity score matching. Thyroid 23: 1408-1415, 2013.

32. Pacini F: Management of papillary thyroid microcarcinoma: primum non nocere! J Clin Endocrinol Metab 98: 1391-1393, 2013.

33. Ito $\mathrm{Y}$, Tomoda $\mathrm{C}$, Uruno $\mathrm{T}$, et al: Papillary microcarcinoma of the thyroid: how should it be treated? World J Surg 28: 1115-1121, 2004

34. Ito Y, Uruno T, Nakano K, et al: An observation trial without surgical treatment in patients with papillary microcarcinoma of the thyroid. Thyroid 13: 381-387, 2003.

35. Sugitani I and Fujimoto Y: Symptomatic versus asymptomatic papillary thyroid microcarcinoma: a retrospective analysis of surgical outcome and prognostic factors. Endocr J 46: 209-216, 1999.
36. Yu XM, Wan Y, Sippel RS and Chen H: Should all papillary thyroid microcarcinomas be aggressively treated? An analysis of 18,445 cases. Ann Surg 254: 653-660, 2011.

37. Hugo H, Ackland ML, Blick T, et al: Epithelial - mesenchymal and mesenchymal - epithelial transitions in carcinoma progression. J Cell Physiol 213: 374-383, 2007.

38. Vasko V, Espinosa AV, Scouten W, et al: Gene expression and functional evidence of epithelial-to-mesenchymal transition in papillary thyroid carcinoma invasion. Proc Natl Acad Sci USA 104: 2803-2808, 2007.

39. Zeisberg M and Neilson EG: Biomarkers for epithelial-mesenchymal transitions. J Clin Invest 119: 1429-1437, 2009.

40. Knauf JA, Sartor MA, Medvedovic M, et al: Progression of BRAF-induced thyroid cancer is associated with epithelialmesenchymal transition requiring concomitant MAP kinase and TGF $\beta$ signaling. Oncogene 30: 3153-3162, 2011.

41. Virk RK, Van Dyke AL, Finkelstein A, et al: BRAFV600E mutation in papillary thyroid microcarcinoma: a genotypephenotype correlation. Mod Pathol 26: 62-70, 2013.

42. So YK, Son YI, Baek CH, et al: Expression of sodium-iodide symporter and TSH receptor in subclinical metastatic lymph nodes of papillary thyroid microcarcinoma. Ann Surg Oncol 19: 990-995, 2012.

43. Kim HJ, Kim NK, Choi JH, et al: Radioactive iodine ablation does not prevent recurrences in patients with papillary thyroid microcarcinoma. Clin Endocrinol (Oxf) 78: 614-620, 2012. 